\title{
论 文
}

\section{基于单元䢃裂法的全耦合水力压裂数值模拟}

\author{
1. 上海交通大学船舶海洋与建筑工程学院, 上海 200240 ; \\ 2. 中国石油大学石油工程学院, 北京 102249 ; \\ 3. 中石化西北油田分公司, 乌鲁木齐 830011 \\ * E-mail: zhennanzhang@sjtu.edu.cn
}

张振南 ${ }^{1 *}$, 王毓杰 ${ }^{1}$, 牟建业 ${ }^{2}$, 赵兵 ${ }^{3}$, 刘志远 $^{3}$

收稿日期: 2018-04-11; 接受日期: 2018-09-26; 网络版发表日期: 2019-06-05

国家自然科学基金(批准号: 11772190)和“十三五”国家油气重大专项(编号: 2016ZX05014-005-012)资助项目

\begin{abstract}
摘要 单元䢃裂法(element partition method, EPM)是一种裂纹模拟方法, 它利用三角单元的几何性质推导了䢃裂 单元的刚度矩阵. 当有裂纹穿过时, 三角单元转化为䢃裂单元, 可自动地将裂纹面之间的相互作用反映到计算模 型中. 通过这种方式, 可以在背景网格中嵌入任意条裂纹而不需要引入额外自由度, 避免了节理单元的设置及网 格重构. 这使得EPM在大规模节理计算方面具有较强的优势. 为了更有效地模拟水力压裂, 建立了䢃裂单元的全 耦合水-力方程，可同时考虑䢃裂单元内水压对结点力的作用以及结点速度场对渗流场的作用. KGD模型验证结 果表明该方法是有效的. 该方法可以有效地模拟多裂纹水力扩展及汇合过程, 为复杂地层压裂模拟提供了一套简 单、有效的计算方法.
\end{abstract}

关键词单元䢃裂法, 水力压裂, 全耦合方程, 复杂地层, 数值模拟

\section{1 引言}

水力压裂一直是石油增产的一个主要技术手段, 因而能有效地对复杂地层压裂过程进行模拟将对压裂 设计及产量评估具有重要意义. 复杂地层中分布着大 量的节理裂隙，如何有效地将它们反映到计算模型中 却是一个棘手问题. 如果应用传统有限单元法来处理 这一问题，要把这些节理作为内边界进行网格划分. 这种处理方法对于小规模节理裂隙是有效的，而对于 大规模的节理裂隙则很难应用. 扩展有限单元法 $(\mathrm{ex}-$ tended finite element method, XFEM) ${ }^{[1,2]}$ 在这方面较传 统有限单元法有很大优势, XFEM允许裂纹从单元中
穿过. 这样可以在背景网格中直接引入这些节理裂隙, 而不用将它们视为内边界进行网格划分，这使得裂纹 处理大为简便. 近年来, 更有学者将XFEM方法应用于 水力裂纹模拟，取得了很好的进展，例如文献[3 6]等. 但就XFEM方法本身来讲，在处理大规模节理问题时 还存在一定的局限性. 由于采用了结点富集插值技术, XFEM再现了裂纹单元不连续位移场的同时又引入了 额外自由度, 这无疑增加了计算量. 另外, 一般地层中 的节理裂隙处于受压状态，节理面之间的接触摩擦效 应起着非常重要的作用. 而XFEM在处理节理面之间 相互作用时, 却显得有些不足. 为了探索更简单直接 的方法来处理大规模节理问题, 张振南等人 ${ }^{[7,8]}$ 提出了

引用格式: 张振南, 王毓杰, 牟建业, 等. 基于单元䢃裂法的全耦合水力压裂数值模拟. 中国科学: 技术科学, 2019, 49: 716-724

Zhang Z N, Wang Y J, Mou J Y, et al. Hydraulic fracture simulation based on hydro-mechanical coupled element partition method (in Chinese). Sci Sin Tech, 2019, 49: 716-724, doi: 10.1360/N092018-00092 
单元䢃裂法(element partition method, EPM). EPM可以 在背景网格中嵌入大量节理裂隙而不需要网格重构. 节理面之间的接触和摩擦效应会自动反映到计算模型 而无需单独设置接触单元，这为大规模节理计算带来 很大方便. 2010年，文献[9]将这一方法扩展到三维情 况. 但是初始EPM没有考虑䢃裂单元的体变形问题, 在理论上不完备. 为了解决这一问题, Zhang等人 ${ }^{[10]}$ 采 用了特殊局部插值技术考虑了䢃裂单元的体变形问 题. 后来，文献[11,12]又对EPM进行了系列改进和完 善，分别考虑了擘裂单元的裂尖问题和黏结型结构面 问题. 最近, Zhang等人 ${ }^{[13]}$ 又将这一改进EPM扩展到三 维单元. 与XFEM相比, EPM同样允许裂纹从单元中穿 过，但没有引入任何额外自由度，关于二者的本质区 别, 已在文献 $[10,13]$ 中给予详细论述.

近几年, EPM已经用于水力裂纹数值模拟, 如文献 [14 16]等. 但目前EPM没有一个完整的全耦合方程, 还不能考虑裂纹填充效应所消耗的流量. 为此，本文 将在初始EPM基础上建立全耦合方程，发展一套简 单、有效的复杂地层压裂数值模拟方法.

\section{2 单元䢃裂法简介}

单元䢃裂法 ${ }^{[7,8]}$ 基本思想是利用三角单元的几何 特点来建立䢃裂单元(被裂纹穿过的单元)的刚度矩阵 和结点力向量. 如图1所示, 当一个三角单元被裂纹穿 过时, 总有一个结点在裂纹的一侧, 而另外两个结点在 裂纹的另一侧. 在裂纹一侧的一个结点与另外两个结 点可以构成两个接触点对. 基于这两个接触点对可以 推导出三角䢃裂单元的刚度矩阵和结点力向量, 即:

$$
\begin{aligned}
F_{i}= & \frac{L}{2 h}\left[k_{n}\left(u_{6}-u_{2}\right)\left(\delta_{6 i}-\delta_{2 i}\right)\right. \\
& +k_{s}\left(u_{5}-u_{1}\right)\left(\delta_{5 i}-\delta_{1 i}\right) \\
& +k_{n}\left(u_{6}-u_{4}\right)\left(\delta_{6 i}-\delta_{4 i}\right) \\
& \left.+k_{s}\left(u_{5}-u_{3}\right)\left(\delta_{5 i}-\delta_{3 i}\right)\right], \\
K_{i j}= & \frac{L}{2 h}\left[k_{n}\left(\delta_{6 i}-\delta_{2 i}\right)\left(\delta_{6 j}-\delta_{2 j}\right)\right. \\
& +k_{s}\left(\delta_{5 i}-\delta_{1 i}\right)\left(\delta_{5 j}-\delta_{1 j}\right) \\
& +k_{n}\left(\delta_{6 i}-\delta_{4 i}\right)\left(\delta_{6 j}-\delta_{4 j}\right) \\
& \left.+k_{s}\left(\delta_{5 i}-\delta_{3 i}\right)\left(\delta_{5 j}-\delta_{3 j}\right)\right],
\end{aligned}
$$

其中, $F_{i}, u_{i}$ 分别为结点力和结点位移向量的分量,
$\mathbf{F}=\left[F_{1}, \ldots, F_{6}\right]^{\mathrm{T}}, \mathbf{u}=\left[u_{1}, \ldots, u_{6}\right]^{\mathrm{T}} ; L$ 为䢃裂单元中节理 的长度; $h$ 为节理厚度; $k_{n}, k_{s}$ 分别为节理面的法向和切 向刚度系数; $\delta_{i j}$ 为Kronecker符号.

在应用该方法时, 直接对裂纹体(图2(a))进行网格 划分, 而不考虑裂纹体的几何完整性(图2(b)). 当网格 划分完后，根据节理和单元坐标判断那些单元被裂纹 穿过(图2(c)). 对于被裂纹穿过的单元调用䢃裂单元刚 度矩阵, 而对于其他单元则调用一般单元刚度矩阵. 通 过这种方法, 可以直接在背景网格中引入大量裂纹, 而 不需要增加任何自由度. 由于这种初始EPM没有考虑 单元的体变形问题, 因而在同样网格大小的条件下没 有XFEM的计算精度高, 但却较XFEM简单、直接, 能 够有效地模拟节理的张开、接触和摩擦行为. 当单元 尺寸逐渐变小时, 由于忽略单元体变形而引起的误差 会逐渐变小, 这使得初始EPM的计算精度会提高. 在 后续发展的改进型EPM ${ }^{[10,11,13]}$ 中由于采用特殊局部插 值技术考虑了䢃裂单元的体变形问题，在粗网格情况 下其计算精度大为改善.

\section{3 䢃裂单元的渗流方程}

\section{1 等效渗透系数}

当单元中有一裂纹穿过时, 其渗流能力来自两方

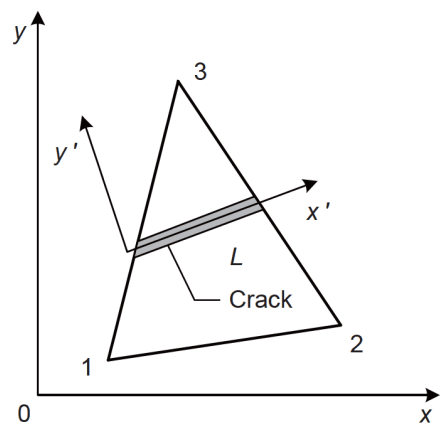

图 1 被裂纹穿过的三角单元
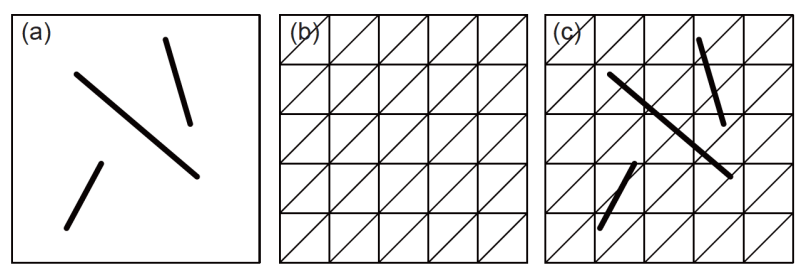

图 2 单元䢃裂法实现过程. (a) 裂纹体; (b) 对裂纹体进行网 格划分而不考虑裂纹的存在; (c) 裂纹与部分单元相割 
面：一为单元基质本身的渗透性，二为裂纹的渗透性. 前者由基质材料的渗透系数决定; 而后者则需要引入 不同的渗透法则. 当裂隙中的流场为层流时，其渗流 行为可由立方定律来描述, 即:

$q=-\frac{a^{2}}{12 \mu} \cdot A_{\mathrm{f}} i$,

其中, $q$ 为穿过裂纹的流量; $a$ 为裂纹张开度; $A_{\mathrm{f}}$ 为水流 通过的面积, 在二维裂纹中 $A_{\mathrm{f}}=a ; i$ 为水力梯度; $\mu$ 为流 体黍性系数.

在模拟水力裂纹过程中，若单独考虑裂纹将会增 加很多不便. 如果将裂纹弥散到整个单元，通过引入 基质等效渗透系数来反映裂纹的导流能力，则使问题 简单化. 为了达到这一目的，需在式(2)基础上导出辟 裂单元的等效渗透系数. 假设一正四边形(图3)有一条 裂纹穿过时，令用于反映裂纹导流能力的基质等效渗 透系数为 $k_{\mathrm{f}}$. 在水力梯度 $i$ 作用下，通过等效基质的流 量为 $q=-k_{\mathrm{f}} / \mu \cdot A i$ (假设满足达西定律). 在同样的水力 梯度作用下, 通过裂纹的流量为 $q=-a^{3} /(12 \mu) \cdot i$. 将两 者流量相等, 即: $-k_{\mathrm{f}} / \mu \cdot A i=-a^{3} /(12 \mu) \cdot i$, 即可得出䢃 裂单元的等效渗透系数为

$k_{\mathrm{f}}=\frac{a^{3}}{12 A}$,

其中, $A$ 在数值上等于正四边形的边长, 在此可以理解 为单元的特征通量面积. 对于非规则几何形状, $A$ 取不 同数值, 其大小与单元尺寸相关, 即:

$A=c V^{1 / 2}$,

其中, $V$ 为单元面积; $c$ 为单元几何系数, 取决于单元规 则度, 对于正四边形 $c=1$, 而对于其他形状(如三角形单 元)则需要进行标定.

结合式(3)和(4), 䢃裂单元的渗透系数可写为

$k=k_{\mathrm{m}}+k_{\mathrm{f}}=k_{\mathrm{m}}+\frac{a^{3}}{12 c \sqrt{V}}$,

其中, $k_{\mathrm{m}}$ 为基质渗透系数. 在实际计算中, 裂纹的张开 度可取两接触点对的平均值, 即:

$a=\frac{1}{2}\left[\begin{array}{llll}0,-1, & 0,-1,0,2\end{array}\right] \mathbf{T} \mathbf{u}$,

其中, $\mathbf{T}$ 为整体坐标到裂纹局部坐标的转换矩阵(如 图1); $\mathbf{u}$ 为三角单元的结点位移向量.

式(6)实际上只考虑了结点法向位移引起的裂纹

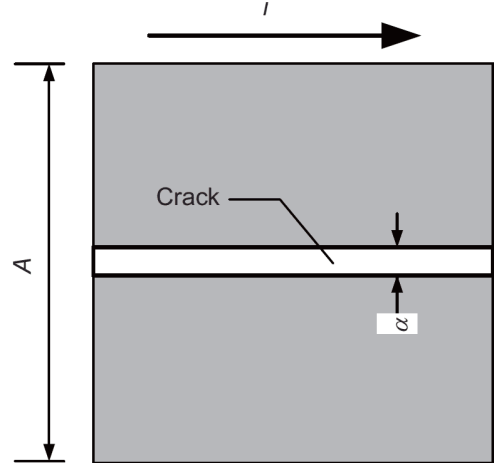

图 3 水力梯度作用下的含有中间贯穿性裂纹的正四边形

张开度. 而对于岩体节理, 由于节理面是粗䊁的, 剪切 位移也会导致张开度增大, 即: 剪胀. 但剪胀幅度受制 于多种因素，如节理强度、法向应力及剪切位移的大 小等. 为了突出主要建模思想，本文暂且不考虑剪胀 对裂纹张开度的影响.

立方定律成立的前提是裂缝壁面平行光滑，缝宽 不能太大, 水流应满足层流条件. 但在现实中, 节理壁 面很粗龯, 且不平行. 因此, 很难对于整条节理采用立 方定律进行分析. 如果将这种节理进行分割, 离散成较 短的单元段, 在局部小单元段内可以近似地认为节理 面是平行光滑的，又考虑到常规水力压裂中，水在裂 隙中的流速并不是很大，可以近似地认为是层流，因 而对于小单元段可以采用立方定律. 本文所述的单元 䢃裂法实质上就是将长节理离散为很多䢃裂单元，每 个䢃裂单元即为小单元段，在小单元段内采用立方定 律, 这样模拟出的结果具有很高的计算精度, 这一点 已在文献[17]中有所证实. 为了反映整条节理的粗粘 度和张开度的变化, 可以根据真实情况或统计规律赋 予每个䢃裂单元(小单元段)以不同的初始张开度，这 一方法已在文献[18]中有所应用, 起到了很好的效果.

\section{2 渗透矩阵}

连续介质的渗流方程为

$k \nabla^{2} P+q=s \cdot \frac{\partial P}{\partial t}$.

将式(7)进行有限元离散, 可得矩阵形式的单元渗流 方程:

$\mathbf{K P}+\mathbf{S} \dot{\mathbf{P}}=\mathbf{Q}$,

其中, $\mathbf{P}, \dot{\mathbf{P}}$ 分别为单元结点水压力场及其一阶导数; $\mathbf{K}$ 
为渗透系数矩阵; $\mathrm{S}$ 为咜水系数矩阵; $\mathrm{Q}$ 为结点流量 向量.

\section{3 等效渗透系数有效性验证}

为了验证等效渗透系数(式(5))的有效性, 进行模 拟验证. 模拟试件为一正方形域, 如图4(a)所示. 在试 件中间预置一条水平贯穿性裂纹，并给定裂纹张开度 为 $a=0.1 \mathrm{~mm}$. 正方形左右两侧分别施加 1 和 $0 \mathrm{MPa}$ 水 压，上下边界为不透水边界. 如果将基质渗透系数取 很小，则通过基质的流量可以忽略不计．在水压梯度 作用下，通过正方形计算域的流量可由立方定律计算 得到, 并将这一值作为理论值. 然后, 采用随机三角形 网格对正方形计算域进行离散模拟. 当单元几何系数 取 $c=1$ 时, 所模拟的流量与理论值对比如图4(b)所示. 从图4(b)可看出, 在不同尺寸网格条件下, 模拟流量值 与理论值基本平行, 这说明计算结果是稳定的, 对单元 尺寸并不敏感. 根据模拟与理论值对比, 对于三角单元 取几何系数 $c=1.5294$, 再重新模拟这一算例, 所得的模 拟结果与理论值对比如图4(c)所示. 从图中可看出二 者结果基本重合，随着网格变小，模拟值的相对误差 在 $0.5 \%$ 以内，如图 $4(\mathrm{~d})$ 所示. 这说明等效渗透系数(式

(a)
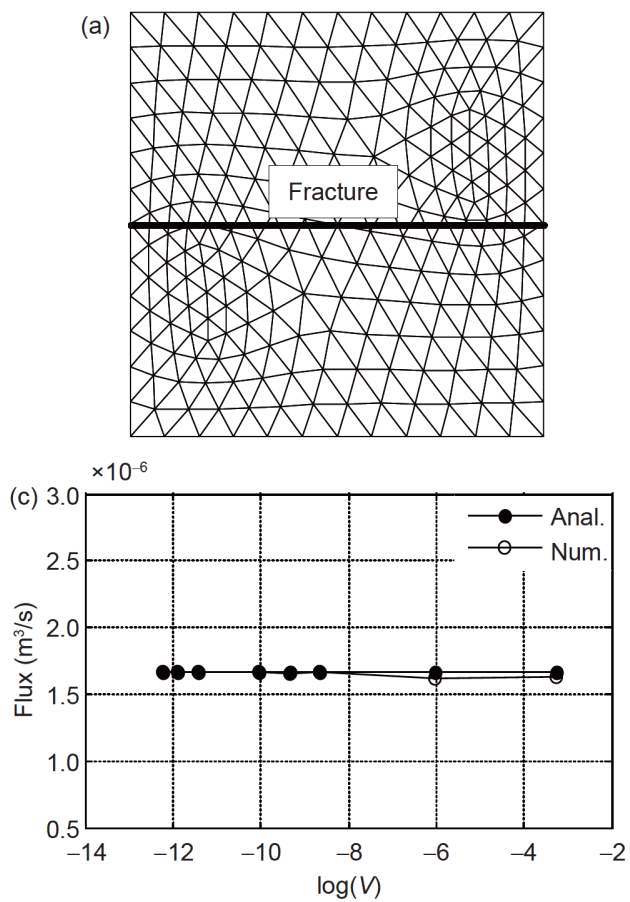

(5))是正确的, 对于三角单元可取几何系数为 $c=1.5294$.

\section{4 䢃裂单元全耦合方程}

当外界向繴裂单元注入流量时，液体除了渗透滤 失外，还有一部分用于填充裂纹(图5). 䢃裂单元中的 裂纹体积可计算为

$$
\begin{aligned}
& V=\frac{\left(u_{6}^{\prime}-u_{2}^{\prime}+u_{6}^{\prime}-u_{4}^{\prime}\right) L}{2} \\
& =\frac{L}{2}\left[\begin{array}{llllll}
0, & -1, & 0, & -1, & 0, & 2
\end{array}\right] \mathbf{T} \mathbf{u},
\end{aligned}
$$

其中, $u_{i}^{\prime}(i=1,2,3,4,5,6)$ 为䢃裂单元局部坐标系下的结 点位移; $L$ 为䢃裂单元内裂纹长度.

用于裂纹填充所消耗的流量可表示为

$$
\begin{aligned}
q_{f} & =\frac{\mathrm{d} V}{\mathrm{~d} t} \\
& =\frac{L}{2}\left[\begin{array}{lllll}
0, & -1, & 0, & -1, & 0,
\end{array}\right] \mathbf{T} \dot{\mathbf{u}},
\end{aligned}
$$

其中, $\dot{\mathbf{u}}$ 为整体坐标系下三角单元结点速度向量.

如果将总填充流量平均分配到䢃裂单元的 3 个结 点, 则结点流量与速度的关系为
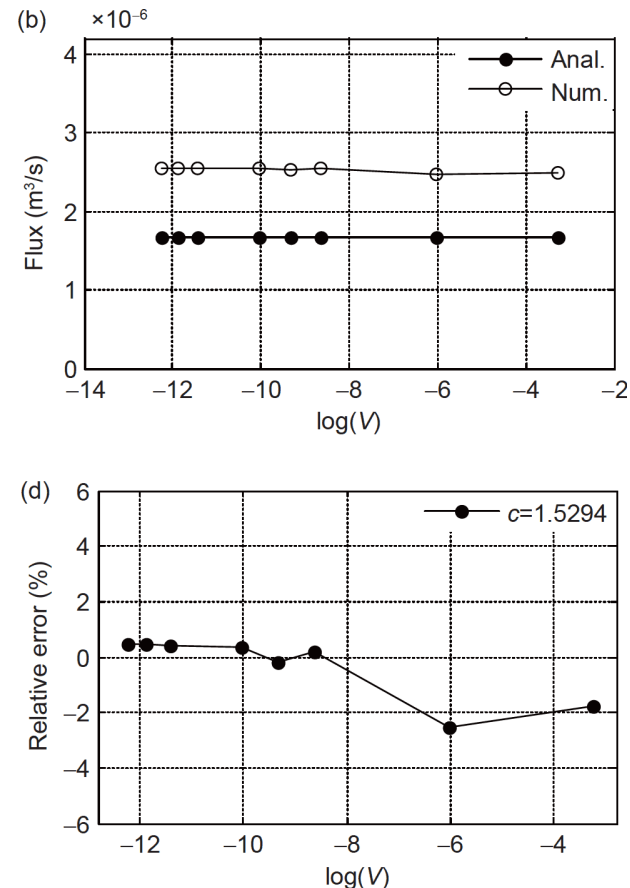

图 4 䢃裂单元等效渗透系数验证. (a) 含贯穿性裂纹的正方形计算域网格划分; (b) 取单元几何系数 $c=1$ 时模拟流量与理论值 对比; (c) 取单元几何系数 $c=1.5294$ 时模拟流量与理论值对比; (d) 相对误差 


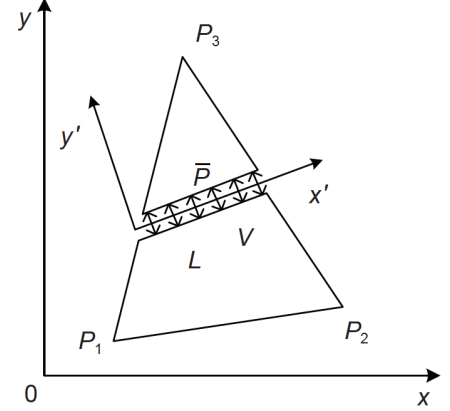

图 5 幦裂单元中的裂纹体积和平均水压

$\mathbf{q}=\lambda[\dot{\mathbf{u}}]$,

其中, $\lambda$ 为结点速度对流量的作用矩阵,

$\lambda=\frac{L}{6}\left[\begin{array}{llllll}0 & -1 & 0 & -1 & 0 & 2 \\ 0 & -1 & 0 & -1 & 0 & 2 \\ 0 & -1 & 0 & -1 & 0 & 2\end{array}\right] \mathbf{T}$.

由式(8)和(11)可得出考虑结点速度场作用的渗流方 程为

$\mathbf{K P}+\mathbf{S} \dot{\mathbf{P}}+\lambda \dot{\mathbf{u}}=\mathbf{Q}$.

液体进入裂纹后会对裂纹面产生压力(图5), 从而 产生附加结点力. 裂纹内平均水压可计算为 $\bar{P}=$ $\left(P_{1}+P_{2}+P_{3}\right) / 3$. 在裂纹局部坐标系内, 由平均水压所产 生的等效结点力为

$\mathbf{R}^{\prime}=\frac{1}{2}\left[\begin{array}{llllll}0, & -1, & 0, & -1, & 0, & 2\end{array}\right]^{\mathrm{T}} L \bar{P}$.

因此，整体坐标系下水压所产生的等效结点力与 结点水压的关系为

$\mathbf{R}=\mathbf{B P}$,

其中, $\mathrm{B}$ 为结点水压对结点力的作用矩阵,

$\mathbf{B}=\frac{L}{6} \mathbf{T}^{\mathbf{T}}\left[\begin{array}{ccc}0 & 0 & 0 \\ -1 & -1 & -1 \\ 0 & 0 & 0 \\ -1 & -1 & -1 \\ 0 & 0 & 0 \\ 2 & 2 & 2\end{array}\right]$.

由式(14)可得出考虑水压的动力学方程为

$\mathbf{M} \ddot{\mathbf{u}}+\mathbf{C} \dot{\mathbf{u}}+\mathbf{F}(\mathbf{u})-\mathbf{B P}=\mathbf{R}$,

其中, $\mathrm{M}$ 为结点质量矩阵, $\mathrm{C}$ 为结点阻尼矩阵. 对比式 (12)及式(15), 发现矩阵 $\lambda$ 与矩阵 $\mathbf{B}$ 互为转置关系, 即:
$\mathbf{B}=\lambda^{\mathrm{T}}$. 从而由式(13)和(16)可得䢃裂单元的全耦合方 程为

$$
\begin{aligned}
& \mathbf{M u ̈}+\mathbf{C} \dot{\mathbf{u}}+\mathbf{F}(\mathbf{u})-\mathbf{B P}=\mathbf{R}, \\
& \mathbf{B}^{\mathrm{T}} \dot{\mathbf{u}}+\mathbf{S} \dot{\mathbf{P}}+\mathbf{k P}=\mathbf{Q} .
\end{aligned}
$$

在水力驱动下, 裂纹要发生扩展. 至于裂纹何时及 如何发生扩展可由相应的断裂准则来确定. 当裂纹发 生扩展时, 根据裂纹扩展长度及角度来判断哪些单元 被新扩展裂纹切割并计算譬裂单元的虚结点坐标. 被 切割的单元则由完整单元转变为䢃裂单元, 如图 6 所示.

\section{5 数值求解方案}

在数值模拟过程中, 采用中心差分法求解式(17). 在中心差分法中，相邻时间步 $\left(t_{0}\right.$ 和 $\left.t_{1}\right)$ 之间的场量关 系为

$$
\begin{aligned}
& \dot{\mathbf{u}}_{1}=\frac{1}{\theta \Delta t} \mathbf{u}_{1}-\frac{1}{\theta \Delta t} \mathbf{u}_{0}-(1 / \theta-1) \dot{\mathbf{u}}_{0}, \\
& \ddot{\mathbf{u}}_{1}=\frac{1}{\theta \Delta t} \dot{\mathbf{u}}_{1}-\frac{1}{\theta \Delta t} \dot{\mathbf{u}}_{0}-(1 / \theta-1) \ddot{\mathbf{u}}_{0} .
\end{aligned}
$$

由式(18)对式(17)进行时间域离散可得

$$
\begin{aligned}
& {\left[\begin{array}{c}
\left.\frac{1}{\theta^{2} \Delta t^{2}} \mathbf{M} \mathbf{u}_{1}+\frac{1}{\theta \Delta t} \mathbf{C} \mathbf{u}_{1}+\mathbf{F}_{1}\left(\mathbf{u}_{1}\right)-\mathbf{B} \mathbf{P}_{1}\right] \\
-\mathbf{B}^{\mathrm{T}} \mathbf{u}_{1}+\left(-\mathbf{S}-\theta \Delta t \mathbf{k}_{1}\right) \mathbf{P}_{1}
\end{array}\right]} \\
& =\left[\begin{array}{c}
\mathbf{R}_{1}+(1 / \theta-1) \mathbf{R}_{0} \\
-\theta \Delta t \mathbf{Q}_{1}-\Delta t(1-\theta) \mathbf{Q}_{0}
\end{array}\right] \\
& +\left[\begin{array}{c}
\frac{1}{\theta^{2} \Delta t^{2}} \mathbf{M u}_{0}+\frac{1}{\theta^{2} \Delta t} \mathbf{M u}_{0}-(1 / \theta-1) \mathbf{F}_{0}\left(\mathbf{u}_{0}\right) \\
\Delta t(1-\theta) \mathbf{k}_{0} \mathbf{P}_{0}
\end{array}\right] \\
& +\left[\begin{array}{c}
\frac{1}{\theta \Delta t} \mathbf{C u}_{0}+(1 / \theta-1) \mathbf{B} \mathbf{P}_{0} \\
-\mathbf{B}^{\mathrm{T}} \mathbf{u}_{0}-\mathbf{S P}_{0}
\end{array}\right] .
\end{aligned}
$$

对式(19)可采用Newton-Raphson法进行迭代求解, 其迭代格式为
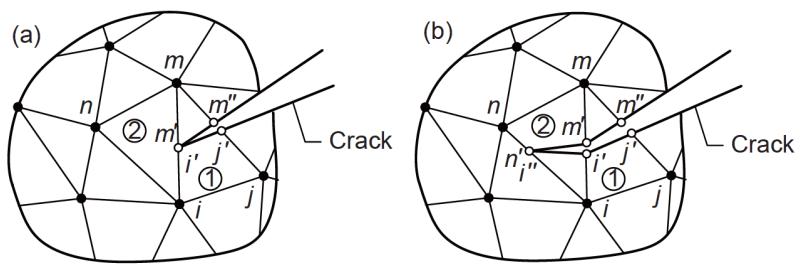

图 6 裂纹扩展过程. (a) 裂纹扩展前; (b) 裂纹扩展后 


$$
\begin{aligned}
& {\left[\begin{array}{cc}
\frac{1}{\theta^{2} \Delta t^{2}} \mathbf{M}+\frac{1}{\theta \Delta t} \mathbf{C}+\mathbf{F}_{1}^{\prime}\left(\mathbf{u}_{1}^{i}\right), & -\mathbf{B} \\
-\mathbf{B}^{\mathrm{T}}, & -\mathbf{S}-\theta \Delta t \mathbf{k}_{1}
\end{array}\right]\left[\begin{array}{l}
\Delta \mathbf{u}_{1}^{i+1} \\
\Delta \mathbf{P}_{1}^{i+1}
\end{array}\right]} \\
& =\left[\begin{array}{c}
\mathbf{R}_{1}+(1 / \theta-1) \mathbf{R}_{0} \\
-\theta \Delta t \mathbf{Q}_{1}-\Delta t(1-\theta) \mathbf{Q}_{0}
\end{array}\right] \\
& +\left[\begin{array}{c}
\frac{1}{\theta^{2} \Delta t^{2}} \mathbf{M} \mathbf{u}_{0}+\frac{1}{\theta^{2} \Delta t} \mathbf{M} \dot{\mathbf{u}}_{0}-(1 / \theta-1) \mathbf{F}_{0}\left(\mathbf{u}_{0}\right) \\
\Delta t(1-\theta) \mathbf{k}_{0} \mathbf{P}_{0}
\end{array}\right] \\
& +\left[\begin{array}{cc}
\frac{1}{\theta \Delta t} \mathbf{C u}_{0}+(1 / \theta-1) \mathbf{B} \mathbf{P}_{0} \\
-\mathbf{B}^{\mathrm{T}} \mathbf{u}_{0}-\mathbf{S P}_{0}
\end{array}\right] \\
& {\left[\begin{array}{c}
\frac{1}{\theta^{2} \Delta t^{2}} \mathbf{M u}_{1}^{i}+\frac{1}{\theta \Delta t} \mathbf{C u}_{1}^{i}+\mathbf{F}_{1}\left(\mathbf{u}_{1}^{i}\right)-\mathbf{B} \mathbf{P}_{1}^{i} \\
-\mathbf{B}^{\mathrm{T}} \mathbf{u}_{1}^{i}-\mathbf{S} \mathbf{P}_{1}^{i}-\theta \Delta t \mathbf{k}_{1}^{i} \mathbf{P}_{1}^{i}
\end{array}\right],} \\
& {\left[\begin{array}{l}
\mathbf{u}_{1}^{i+1} \\
\mathbf{P}_{1}^{i+1}
\end{array}\right]=\left[\begin{array}{l}
\mathbf{u}_{1}^{i} \\
\mathbf{P}_{1}^{i}
\end{array}\right]+\left[\begin{array}{l}
\Delta \mathbf{u}_{1}^{i+1} \\
\Delta \mathbf{P}_{1}^{i+1}
\end{array}\right] \text {. }}
\end{aligned}
$$
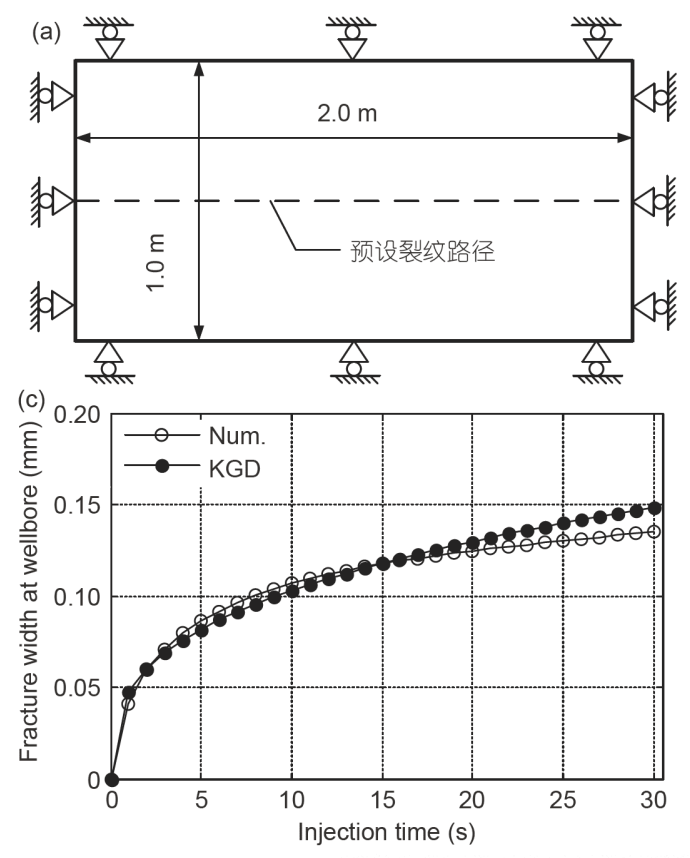

(b)

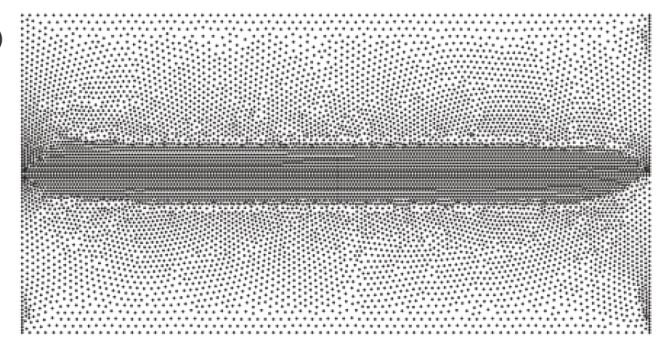

(d)

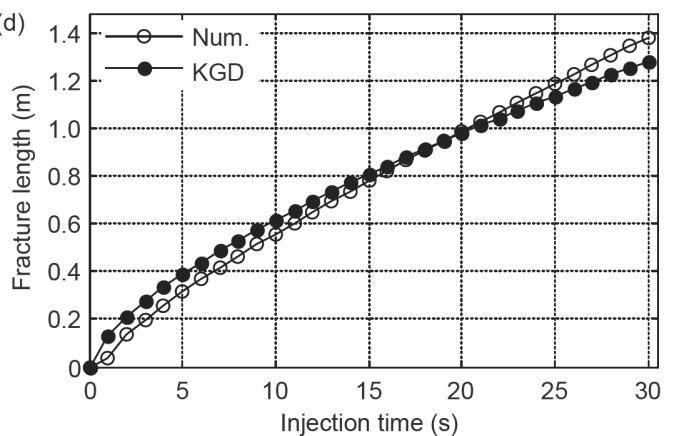

其中, $L$ 为裂纹扩展长度; $w$ 为井口处裂纹张开度; $G$ 为 材料剪切模量; $v$ 为材料泊松比; $Q$ 为井口注入流量; $\mu$ 为流体黏性系数; $t$ 为流体注入时间.

验证模型为一长方形计算域(图7(a)), 为了避免裂 纹路径网格依赖性给计算结果带来的误差, 在计算域

(e)

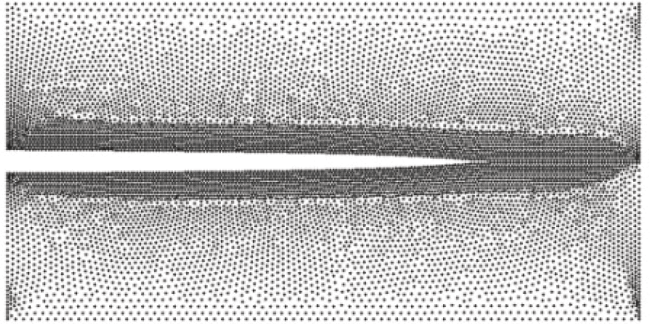

图 7 计算模型的KGD验证. (a) 计算域及边界条件; (b) 网格划分; (c) 井口处张开度与时间关系; (d) 裂纹长度与时间关系; (e) 变形后的网格(30 s, 放大 200 倍) 
中间预设一条水平向贯穿型裂纹路径. 计算采用三角 单元网格(图7(b))单元总数为 18682 , 结点总数为 9493 . 被预设路径切割的单元为潜在䢃裂单元. 这些潜在䢃 裂单元在未达到开裂准则前仍然按完整单元进行计 算. 一旦达到开裂条件时, 潜在䢃裂单元被激活成为䢃 裂单元. 为了表示井口, 在计算域左端处事先激活两个 潜在䢃裂单元作为井口, 并在相应结点上施加流量, 井 口处裂纹张开度可由位于井口两侧的结点相对位移进 行计算. 计算参数为: 杨氏模量 $E=40.0 \mathrm{GPa}$, 泊松比 $v=0.2$, 流体黏性系数 $\mu=0.05 \mathrm{~Pa} \mathrm{~s}$ ，基质渗透系数

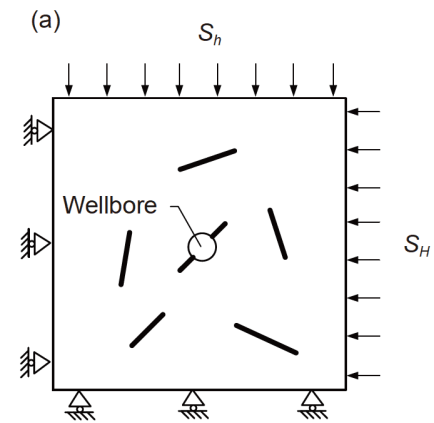

(c)

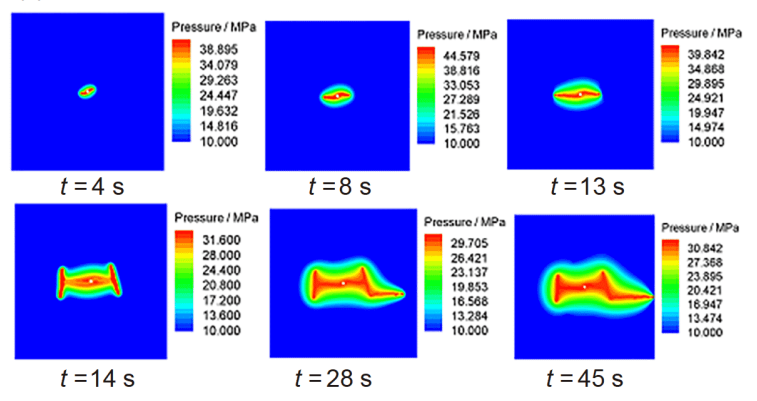

$k=1.0 \times 10^{-18} \mathrm{~m}^{2}$, 注入流量为 $5.0 \times 10^{-6} \mathrm{~m}^{3} / \mathrm{s}$.

所模拟的井口张开度及裂纹扩展长度与时间关系 如图7(c)和(d)所示, 裂纹扩展如图7(e)所示. 从图中可 看出, 所模拟的结果与KGD模型理论结果基本一致, 这说明本模型是有效的.

\section{7 算例}

模拟算例如图8(a)所示，在方形计算域 $(1 \mathrm{~m} \times 1 \mathrm{~m})$ 中心有一井口(直径 $R=0.01 \mathrm{~m})$. 井周分布 5 条裂纹. 水

(b)

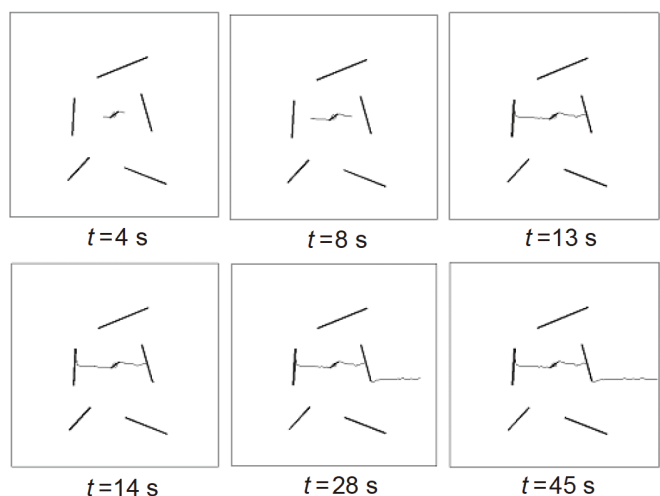

(d)
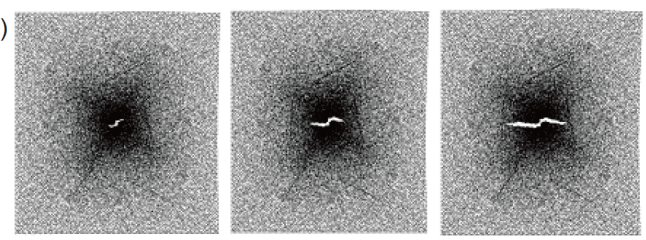

$t=4 \mathrm{~s}$

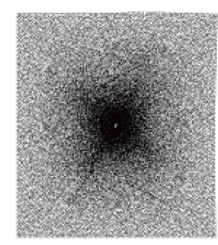

$t=14 \mathrm{~s}$

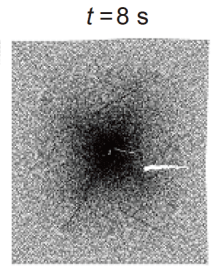

$t=13 \mathrm{~s}$

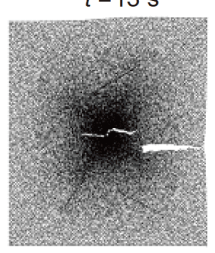

$t=45 \mathrm{~s}$

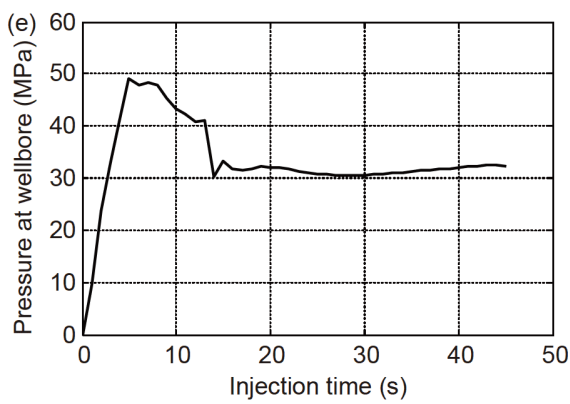

图 8 多裂纹水力压裂模拟. (a) 计算域(边长为 $1 \mathrm{~m}$ 的正方形, 内部分布 5 条裂纹, 在井口处有一倾角 $45^{\circ}$ 、长度为 $0.014 \mathrm{~m}$ 的对 称射孔); (b) 水力裂纹扩展过程; (c) 水压分布图; (d) 网格变形图(结点位移放大100倍); (e) 井口压力时程曲线 
平和坚向地应力分别为 $S_{\mathrm{H}}=50.0 \mathrm{MPa}, S_{\mathrm{h}}=25.0 \mathrm{MPa}$. 计 算参数为: 杨氏模量 $E=40.0 \mathrm{GPa}$, 泊松比 $\nu=0.2$, 流体黏 性系数 $\mu=0.05 \mathrm{~Pa} \mathrm{~s}$, 裂纹面法向刚度系数 $k_{n}=10^{3} \mathrm{GPa}$, 切向刚度系数 $k_{s}=1.0 \mathrm{kPa}$; 基质渗透系数 $k=1.0$ $\times 10^{-15} \mathrm{~m}^{2}$, 注入流量 $1.0 \times 10^{-5} \mathrm{~m}^{3} / \mathrm{s}$.

在裂纹判断方面, 可以有很多断裂准则供选取. 本 文采用应变能密度(strain energy density, SED)准 则 ${ }^{[20,21]}$. 选取SED准则的原因是针对岩石材料SED能 综合考虑裂纹尖端附近应力和应变分量的作用，能够 较好地处理复合型裂纹扩展问题, 同时在有限元计算 上较容易实现．当应变能密度因子 $S$ 超过其临界值 $S_{\mathrm{cr}}$ 时 $\left(S>S_{\mathrm{cr}}\right)$, 裂纹开裂, 本文取 $S_{\mathrm{cr}}=15.0 \mathrm{~N} / \mathrm{m}$. 其开裂方向 为即应变能密度因子最小的方向, 即沿 $\mathrm{d} S / \mathrm{d} \theta=0$ 方向. 模拟结果如图8(b) (e)所示. 图8(b)为不同时刻水力裂 纹扩展图. 从图中可看出随着注入时间的增加, 裂纹 开始起裂、扩展并与已有裂纹汇合．当水力裂纹与已 有裂纹汇合后，已有裂纹又开始扩展(如图8(b), $t=28 \mathrm{~s}$ 时刻). 相应的水压分布如图8(c)所示. 裂纹张开度如 图8(d)所示. 值得注意的是当 $t=13 \mathrm{~s}$ 时, 裂纹是张开的;
而当 $t=14 \mathrm{~s}$ 时裂纹突然“闭合”，然后随着流量注入，又 慢慢张开. 这是由于在 $t=14 \mathrm{~s}$ 时, 水力裂纹突然与已有 裂纹贯穿, 导致水突然流入到已有裂纹, 使水压突然下 降所致. 从图8(e)的压力时程曲线也能得到验证, 即: 在 $t=14 \mathrm{~s}$ 时水压突然有一很大波动. 这一点也从另一 角度证明了本文所推导的全耦合方程的能够反映力学 场与渗流场之间的相互作用.

\section{8 结论}

在初始单元䢃裂法的基础上建立了水-力全耦合 方程. 通过该方程可以有效地考虑䢃裂单元内水压对 结点力的作用以及结点速度场对渗流场的作用. 通过 KGD模型的验证, 说明所建立的全耦合方程是有效的. $\mathrm{EPM}$ 在不引入任何额外自由度的情况下允许裂纹从 单元中穿过，因而可以将大规模节理嵌入到已划好的 背景网格中. 通过所建立力水-力全耦合方程，可以模 拟多裂纹水力扩展，为复杂地层水力压裂模拟提供了 一种简单、有效的方法.

\section{参考文献}

1 Belytschko T, Black T. Elastic crack growth in finite elements with minimal remeshing. Int J Numer Meth Eng, 1999, 45: 601-620

2 Moës N, Dolbow J, Belytschko T. A finite element method for crack growth without remeshing. Int J Numer Meth Eng, 1999, 46: 131-150

3 王涛, 高岳, 柳占立, 等. 基于扩展有限元法的水力压裂大物模实验的数值模拟. 清华大学学报(自然科学版), 2014, 54: 1304-1309

4 曾庆磊, 庄茁, 柳占立, 等. 页岩水力压裂中多簇裂缝扩展的全耦合模拟. 计算力学学报, 2016, 33: 643-648

5 盛茂, 李根生. 水力压裂过程的扩展有限元数值模拟方法. 工程力学, 2014, 31: 123-128

6 Lecampion B. An extended finite element method for hydraulic fracture problems. Commun Numer Meth Eng, 2009, 25: 121-133

7 Zhang Z, Chen Y. Simulation of fracture propagation subjected to compressive and shear stress field using virtual multidimensional internal bonds. Int J Rock Mech Min Sci, 2009, 46: 1010-1022

8 张振南, 陈永泉. 一种模拟节理岩体破坏的新方法: 单元䢃裂法. 岩土工程学报, 2009, 31: 1858-1865

9 黄恺, 张振南. 三维单元䢃裂法与压剪裂纹数值模拟. 工程力学, 2010, 27: 51-58

10 Zhang Z, Wang D, Ge X. A novel triangular finite element partition method for fracture simulation without enrichment of interpolation. Int J Comput Methods, 2013, 10: 1350015

11 张振南, 郑宏, 葛修润. 考虑裂尖点的三角单元䢃裂法. 中国科学: 技术科学, 2013, 43: 1136-1143

12 陈亚雄, 张振南. 基于单元䢃裂法的岩体粘结型结构面数值模拟. 岩土力学, 2013, 34(Suppl 2): 443-447

13 Zhang Z, Wang D, Ge X, et al. Three-dimensional element partition method for fracture simulation. Int J Geomech, 2016, 16: 04015074

14 Zhang Z, Peng S, Ghassemi A, et al. Simulation of complex hydraulic fracture generation in reservoir stimulation. J Pet Sci Eng, 2016, 146: 272285

15 Zhang Z, Ghassemi A. Simulation of hydraulic fracture propagation near a natural fracture using virtual multidimensional internal bonds. Int J Numer Anal Meth Geomech, 2011, 35: 480-495

16 Huang K, Zhang Z, Ghassemi A. Modeling three-dimensional hydraulic fracture propagation using virtual multidimensional internal bonds. Int J Numer Anal Meth Geomech, 2013, 37: 2021-2038 
Peng S, Zhang Z, Li C, et al. Simulation of water flow in fractured porous medium by using discretized virtual internal bond. J Hydrol, 2017, 555: $851-868$

19 Ching H Y. Mechanic of Hydraulic Fracturing. 2nd ed. Houston: Gulf Professional Publishing, 1997. 1-8

20 Kipp M E, Sih G C. The strain energy density failure criterion applied to notched elastic solids. Int J Solids Struct, 1975, 11: 153-173

21 Jeong D Y, Orringer O, Sih G C. Strain energy density approach to stable crack extension under net section yielding of aircraft fuselage. Theor Appl Fract Mech, 1995, 22: 127-137

\title{
Hydraulic fracture simulation based on hydro-mechanical coupled element partition method
}

\author{
ZHANG ZhenNan ${ }^{1}$, WANG YuJie ${ }^{1}$, MOU JianYe ${ }^{2}$, ZHAO Bing $^{3}$ \& LIU ZhiYuan ${ }^{3}$ \\ ${ }^{1}$ School of Naval Architecture, Ocean and Civil Engineering, Shanghai Jiao Tong University, Shanghai 200240, China; \\ ${ }^{2}$ College of Petroleum Engineering, China University of Petroleum, Beijing 102249, China; \\ ${ }^{3}$ SINOPEC Northwest Branch, Urumqi 830011, China
}

Element partition method (EPM) is a fracture simulation method. It takes advantage of geometry feature of triangular element to derive the stiffness matrix of a cracked element. Once a fracture runs across a triangular element, this element will be transformed into a cracked element. The contact and friction between crack faces are automatically incorporated into the numerical model. By this means, any number of fractures can be embedded into a prescribed background mesh without introduction of any extra degree of freedom. It avoids the setup of joint element and remeshing issues in fracture simulation. Hence, the EPM presents great advantage in dealing with the massive fractures. To simulate the hydraulic fracture more effectively, a fully hydro-mechanical coupled equation is established for the cracked element. It can account for the interactions between the mechanical field and the hydraulic field in a cracked element. The KGD verification suggests that this method is validated in hydraulic fracture simulation. It can effectively simulate the multiple hydraulic fracture propagation and coalescence. It provides a simple and efficient simulation method for hydraulic fracturing in the complex reservoir.

element partition method, hydraulic fracture, fully-coupled equation, complex reservoir, numerical simulation doi: $10.1360 /$ N092018-00092 